\title{
ADC by Image Processing
}

\author{
Krisada Vilailak
}

\begin{abstract}
Nowadays, analog meter is still prevalently used because of its accuracy, durability and low-maintenance. However, it is not easy to be taken to do a digital process since reading, saving and putting data into computer are required. Therefore, this research aims at using image processing technique and digital camera to automatically send data from analog meter to computer. It will be done by writing image processing with PHP, and comparing with 30 photos taken by digital camera. After comparing the measurement of data with human reading, it showed least than $0.9 \%$ error.
\end{abstract}

Keywords - analog to digital converter, image processing.

\section{INTRODUCTION}

Analog meter is widely used in laboratories and industrial factories because of its accuracy, durability and low-maintenance. However, the results, nowadays, have to be processed for control and display. Therefore, analog meter is not proper for such operation since taking notes and putting data into computer are required. Moreover, transforming analog meter to be digital meter is complicated, and it costs much.

Thus, this research aims at using image processing technique and digital camera to automatically send data from analog meter to computer. It was expected that the correct results would be equivalent to humans' eyes, and the system would not need to be changed.

\section{DETAIL EXPERIMENTAL}

\section{A. Background Work}

1) Chain Code or Freeman code: Chain code[1] is used to represent a boundary by a connected sequence of straight line segments of specified length and direction. Typically this representation is based on 4 (or) 8 connectivity of the segments (as shown in Fig.1 a-b). The direction of each segment is coded by using a numbering scheme. A boundary code formed as a sequence of such directional numbers is referred to as a Freeman Chain Code. The chain code of a boundary depends on the starting point. Working with code numbers offers a unified way to analyze the shape of the boundary. Chain Code follows the contour in counter clockwise manner and keeps track of the directions as we go from one contour pixel to the next. Fig.1(a) shows 4connectivity and Fig.1(b) shows 8-connectivity of freeman chain code.

Electrical Branch, Faculty of Engineering, Vongchavalitkul University, Nakornratchasrima, Thailand, Email: krisada_vil@vu.ac.th
The main disadvantage of 4-connectivity is that we lose the diagonal points where these points are very useful in most of the image applications. So, in order to overcome the disadvantage of 4-connectivity here we use 8-connectivity. In 8 -connectivity each code can be considered as the angular directions, in multiples of $45^{\circ}$, that we must move to go from one contour pixel to the next.

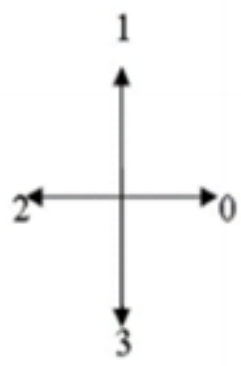

(a)

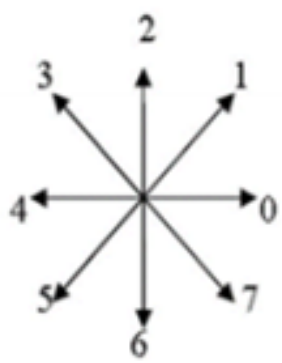

(b)
Fig. 1: Neighbour Directions of Freeman Chain code

2) Chord's Property: The perpendicular bisector of a chord passes through the center of a circle.

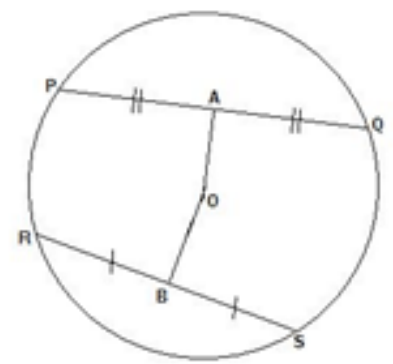

Fig. 2: Circle with 2 chords

In the Fig.2, $O A$ is the perpendicular bisector of the chord $P Q$ and it passes through the center of the circle. $O B$ is the perpendicular bisector of the chord $R S$ and it passes through the center of the circle.

3) The law of cosines:

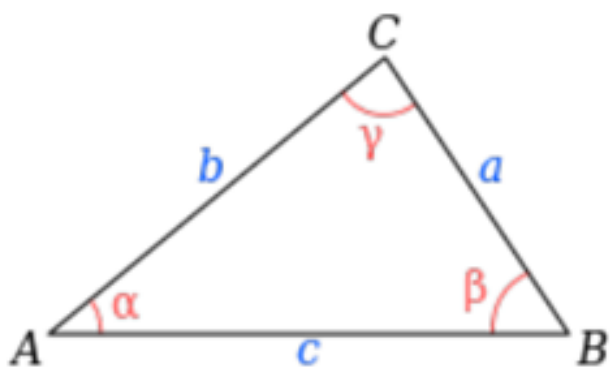

Fig3. Triangle for law of cosine 
In trigonometry, the law of cosines (also known as the cosine formula or cosine rule) relates the lengths of the sides of a triangle to the cosine of one of its angles. Using notation as in Fig.3, the law of cosines states

$$
c^{2}=a^{2}+b^{2}-2 a b \cos \gamma
$$

where $\gamma$ denotes the angle contained between sides of lengths $a$ and $b$ and opposite the side of length $c$.

The law of cosines generalizes the Pythagorean theorem, which holds only for right triangles: if the angle $\gamma$ is a right angle (of measure $90^{\circ}$, or $\pi / 2$ radians), then $\quad \cos \gamma=0$, and thus the law of cosines reduces to the Pythagorean theorem: $c^{2}=a^{2}+b^{2}$

The law of cosines is useful for computing the third side of a triangle when two sides and their enclosed angle are known, and in computing the angles of a triangle if all three sides are known.

By changing which sides of the triangle play the roles of $a, b$, and $c$ in the original formula, the following two formulas also state the law of cosines:

$$
a^{2}=b^{2}+c^{2}-2 b c \cos \alpha \quad \text { and } b^{2}=a^{2}+c^{2}-2 a c \cos \beta
$$

\section{B. Algorithm}

\section{Finding Center of the Circle}

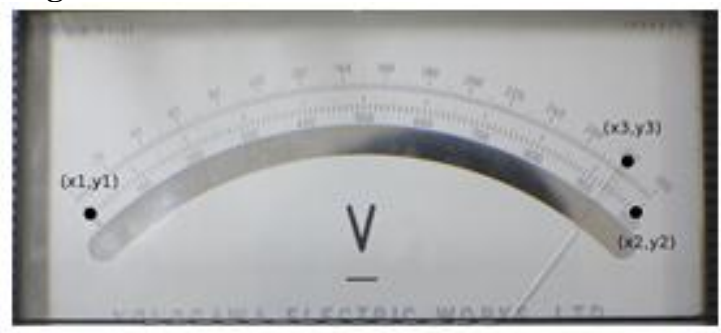

(a)

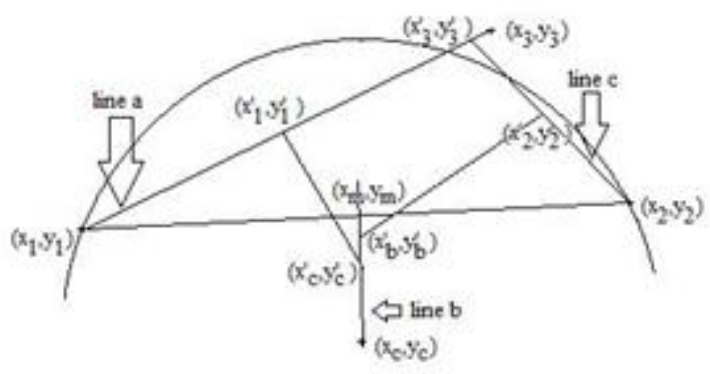

(b)

Fig.3 (a) Input data (b) any point in algorithm

Fig.3(a) shows co-ordinates $\left(x_{1}, y_{1}\right),\left(x_{2}, y_{2}\right),\left(x_{3}, y_{3}\right)$ They derive from taken photo and Freeman chain code, which can be calculated as the following :

1) Equation of straight line a :

$$
\text { calculated slope } \quad m_{1}=\frac{y_{\mathrm{g}}-y_{1}}{x_{\mathrm{g}}-x_{1}}
$$

can give slope $m_{11}$ which is perpendicular with $m_{1}$, according to

$$
m_{11}=-\frac{1}{m_{1}}
$$

2) Equation of straight line $b$ :

$$
\text { -calculated slope } \quad m_{2}=\frac{y_{2}-y_{1}}{x_{2}-x_{1}}
$$

can give slope $m_{22}$ which is perpendicular with $m_{2}$, according to

$$
m_{22}=-\frac{1}{m_{2}}
$$

and find point $\left(x_{\mathrm{m}}, y_{\mathrm{m}}\right)$ in the middle between $\left(x_{1}, y_{1}\right),\left(x_{2}, y_{2}\right)$

$$
\text { , according to } x_{m}=\frac{x_{1}+x_{2}}{2}, y_{m}=\frac{y_{1}+y_{2}}{2}
$$

so Equation of straight line are $\quad y=m_{22} x+b_{2}$

when

....(6)

$$
b_{2}=y_{m}-m_{22} x_{m}
$$

For Chord's Property, it can be seen that center of the circle is in straight line $b$.

3) Marking any point $\left(\mathrm{x}_{3}, \mathrm{y}_{3}\right)$ which is in line a , then

-Finding $\left(x_{1}{ }_{1}, y_{1}{ }_{1}\right)$ in the middle between $\left(x_{1}, y_{1}\right), \quad\left(x_{3}{ }_{3}, y^{\prime}{ }_{3}\right)$ and draw a line to be perpendicular with straight line $a$ through $\left(x_{1}{ }_{1}, y_{1}\right)$ to cross the straight line $b$ at $\left(x^{\prime}, y^{\prime}{ }_{\mathrm{b}}\right)$

-Finding $\left(x_{2}^{\prime}, y_{2}^{\prime}\right)$ in the middle between $\left(x_{2}, y_{2}\right), \quad\left(x_{3}^{\prime}, y_{3}^{\prime}\right)$ and draw a line to be perpendicular with straight line $a$ through $\left(x_{2}^{\prime}, y_{2}^{\prime}\right)$ to cross the straight line $c$ at $\left(x^{\prime}, y^{\prime}{ }_{\mathrm{c}}\right)$

-Changing $\left(x_{3}^{\prime}, y_{3}^{\prime}\right)$ until we got $\left(x_{\mathrm{b}}^{\prime}, y_{\mathrm{b}}^{\prime}\right)=\left(x_{\mathrm{c}}^{\prime}, y_{\mathrm{c}}^{\prime}\right)$, so according to we have $\left(x_{\mathrm{c}}, y_{\mathrm{c}}\right)$ to be the required center of the circle.

-Finding Angle $\theta_{1}, \theta_{2}$ and measuring Value

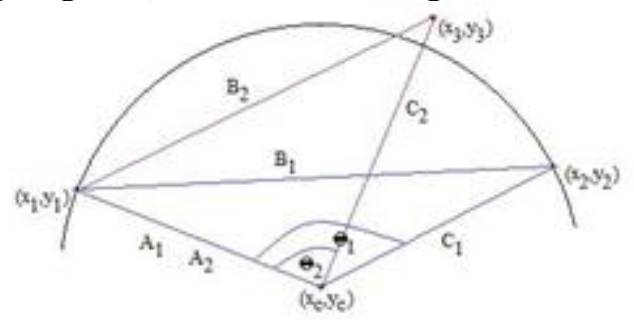

Fig.4 Find angle from law of cosine

4) From fig.4, find distance of $A_{1}, B_{1}, C_{1}, A_{2}, B_{2}, C_{2}$ and for the law of cosines, it can be seen that

$$
\begin{aligned}
& B_{1}^{2}=A_{1}^{2}+C_{1}^{2}-2 A_{1} C_{1} \cos \theta_{1} \text { Then } \theta_{1}=\cos ^{-1} \frac{A_{1}^{2}+C_{1}^{2}-B_{1}^{2}}{2 A_{1} C_{1}} \\
& B_{2}^{2}=A_{2}^{2}+C_{2}^{2}-2 A_{2} C_{2} \cos \theta_{2} \text { Then } \\
& \quad \theta_{2}=\cos ^{-1} \frac{A_{2}^{2}+C_{2}^{2}-B_{2}^{2}}{2 A_{2} C_{2}}
\end{aligned}
$$

5) Finding the ratio of $\frac{\theta_{2}}{\theta_{1}}$, and multiply with full scale value. We will have answer of the required measuring value. Results

\section{RESULTS}

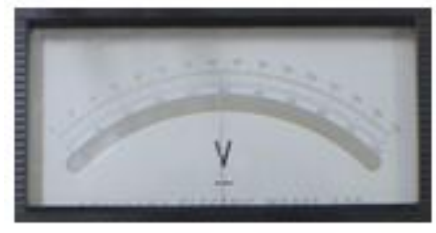

$$
\begin{array}{ll}
\theta_{1} & =89.492 \\
\theta_{2} & =43.543 \\
\text { calc. } & =14.597 \\
\text { Human } & \\
\quad \text { read } & =14.5 \\
\% \text { error } & =0.667
\end{array}
$$



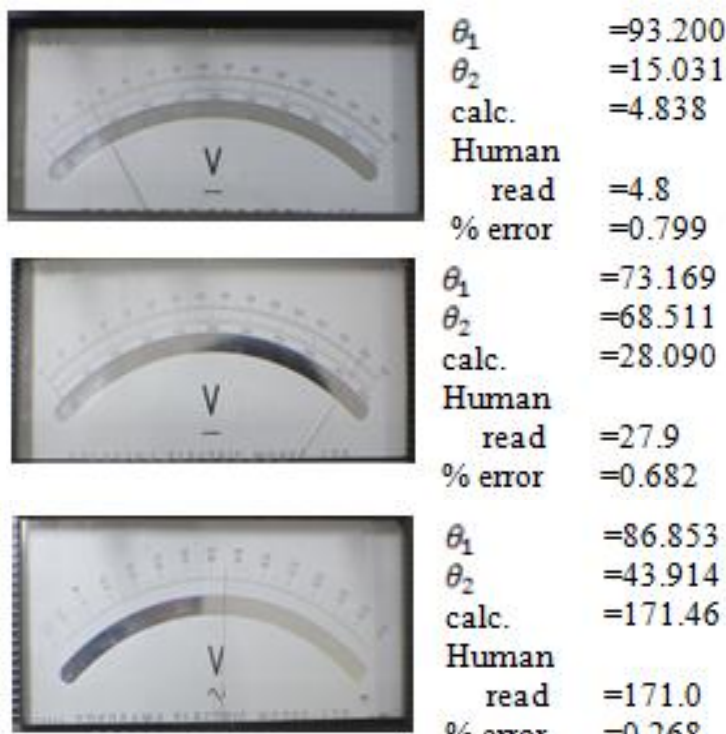

$\theta_{1} \quad=86.853$

$\theta_{2} \quad=43.914$

calc. $\quad=171.46$

Human

read $=171.0$

$\%$ error $\quad=0.268$

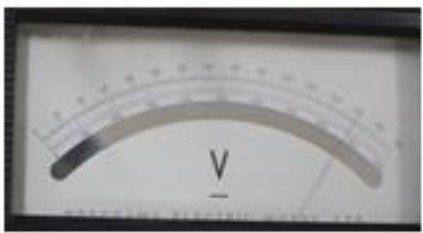

$\theta_{1}=116.93$

$\theta_{2}=102.48$

calc. $=26.294$

Human

read $=26.2$

$\%$ error $=0.358$

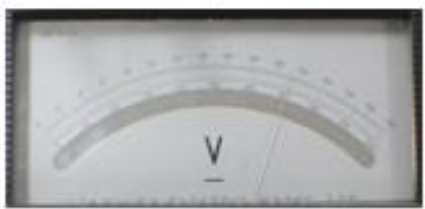

$\begin{array}{ll}\theta_{1} & =89.597\end{array}$

$\theta_{2}=63.564$

calc. $=21.283$

Human

read $=21.2$

$\%$ error $=0.393$

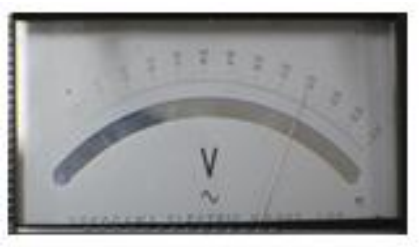

$$
\begin{aligned}
\theta_{1} & =79.365 \\
\theta_{2} & =60.228 \\
\text { calc. } & =242.12 \\
\text { Human } & \\
\text { read } & =240.0 \\
\% \text { error } & =0.887
\end{aligned}
$$

Human

\section{CONCLUSION}

For 30 photos testing, the results were equivalent to humans' reading. It showed less than $0.9 \%$ of error, which can be developed to use with panel meter in industrial factories as well as making untouched data logger for the meter that saves data continuously.

\section{ACKNOWLEDGEMENT}

Without the research presentation fund of Vongchavalitkul University, this research would not have been possible.

\section{REFERENCES}

[1] Digital Image Processing 2nd Edition by Gonzalez and Woods Pearson Publications

[2] http://www.onlinemathlearning.com/chords-circle.html

[3] https://en.wikipedia.org/wiki/Law_of_cosines. 